\title{
Méditerranée
}

Revue géographique des pays méditerranéens / Journal of Mediterranean geography

$127 \mid 2016$

La ségrégation dans les villes de l'Europe méditerranéenne

\section{Una segregazione paradossale e multi-scalare: il caso del quartiere ZEN di Palermo}

A Case of Paradoxical and Multi-Scalar Segregation: The ZEN District of Palermo

Marco Picone

\section{OpenEdition}

Journals

Edizione digitale

URL: http://journals.openedition.org/mediterranee/8389

DOI: 10.4000/mediterranee.8389

ISSN: $1760-8538$

Editore

Presses Universitaires de Provence

\section{Edizione cartacea}

Data di pubblicazione: 1 novembre 2016

Paginazione: 37-46

ISBN: 9791032001370

ISSN: 0025-8296

Questo documento vi è offerto da Université de Valenciennes et du Hainaut-Cambrésis

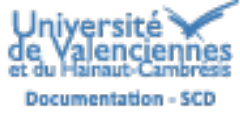

Notizia bibliografica digitale

Marco Picone, " Una segregazione paradossale e multi-scalare: il caso del quartiere ZEN di Palermo »,

Méditerranée [Online], 127 | 2016, Online since 01 November 2016, connection on 23 April 2018. URL

http://journals.openedition.org/mediterranee/8389; DOI : 10.4000/mediterranee.8389 


\title{
Una segregazione paradossale e multi-scalare: il caso del quartiere ZEN di Palermo
}

\author{
A Case of Paradoxical and Multi-Scalar Segregation: The ZEN District of Palermo
}

Marco PICONE

Università degli Studi di Palermo, Dipartimento di Architettura, Faculty Member

marco.picone@unipa.it

\begin{abstract}
Riassunto - L'articolo affronta la questione della segregazione nel quartiere ZEN di Palermo (Sicilia). L'impianto teorico dialoga con la letteratura anglosassone, ma ne prende le distanze nel tentativo di individuare alcune specificità locali del caso. L'obiettivo è duplice: intanto indentificare alcuni "movimenti geostorici" che hanno caratterizzato il quartiere, collegando a ciascuno di questi una fase del processo segregativo; inoltre, rappresentare la segregazione del quartiere in forma cartografica, mediante l'uso di alcuni ideogrammi e mappe mentali.

Parole chiave: segregazione, Europa mediterranea, ZEN, mappe mentali, ideogrammi
\end{abstract}

Il caso dello ZEN, quartiere periferico estremamente noto di Palermo, può fornire indicazioni utili per affrontare la questione della segregazione socio-spaziale nel sud Europa. Come sarà chiaro proseguendo nella lettura, il modello di segregazione in atto allo ZEN mescola alcune caratteristiche della classica ghettizzazione nord-americana ad altre tendenze specifiche delle città mediterranee.

Il presente testo è diviso in 4 sezioni. A seguito di una ricognizione teorica imperniata sull'approccio comparativo, la prima sezione di questo articolo discute alcune questioni relative alla segregazione nelle città mediterranee, nel tentativo di chiarire se lo ZEN possa essere inquadrato come caso tradizionale o, invece, come caso atipico di segregazione. Inoltre, questa prima sezione pone alcune domande fondamentali, cui si darà risposta nelle conclusioni: il fatto che le forme di segregazione sociospaziale urbana siano fortemente mitigate, $\mathrm{o}$ addirittura assenti, è una caratteristica delle città mediterranee? Quali sono i meccanismi che regolano l'esclusione sociale in una città come Palermo? Il neoliberismo e la globalizzazione condizionano in maniera meno forte, rispetto ad altri casi (prevalentemente nord-americani), la polarizzazione sociale della popolazione? Le forme peculiari di segregazione mediterranea seguono logiche spaziali riconoscibili, come per esempio la segregazione verticale? La sezione si conclude dibattendo una tematica nodale e introducendo la proposta più significativa di questo articolo: è possibile ragionare in una prospettiva multi-scalare di segregazione e de-segregazione, a partire dal caso dello ZEN?

La seconda sezione racconta una geostoria del quartiere ZEN: per geostoria si intende di seguito una ricostruzione di alcuni passaggi storicamente significativi, narrata non
Abstract - This article deals with the issue of segregation in the ZEN district of Palermo, Sicily. The theoretical framework is built around Anglo-Saxon references on segregation, but takes its distance in an attempt to identify some local peculiarities of the case study. The article has two main goals: the first is to describe a few 'geohistorical movements' that characterize the district, each connected to a different stage of the segregation processes; the second is to chart these processes through ideograms and mental maps.

Keywords: segregation, Mediterranean Europe, ZEN, mental maps, ideograms

in ordine strettamente cronologico, ma attraverso l'analisi geografica dei luoghi. Vengono selezionati quattro luoghi particolarmente simbolici del quartiere, che sono indicati come "movimenti" geografici e sono proposti come base per riflettere sui cambiamenti che hanno condizionato la periferia palermitana. La sezione fornisce anche alcuni dati quantitativi relativi al quartiere.

Per anni lo ZEN è stato descritto come un "inferno in terra" (FAVA, 2007), schiacciando di fatto le sue reali caratteristiche in un quadro stigmatizzante. La terza sezione, pertanto, prova a stabilire dei criteri metodologici per raccontare la segregazione (o la sua assenza) allo ZEN, proponendo alcune rappresentazioni grafiche e mappe mentali mirate a descrivere in maniera innovativa - benché, naturalmente, solo qualitativa - la segregazione socio-spaziale.

La sezione conclusiva riassume i risultati ottenuti e propone un modello d'interpretazione del caso, per provare a inquadrare epistemologicamente, con un movimento induttivo, le caratteristiche uniche di questa periferia palermitana.

\section{I - Esiste una segregazione mediterranea?}

Condividendo l'approccio post-coloniale agli studi urbani di J. Robinson (2006; 2011), e accogliendo il suo suggerimento di considerare ogni città del mondo come "ordinaria", occorre domandarsi se sia davvero possibile parlare di un modello di città mediterranea, e quali siano le sue caratteristiche. È indubbiamente vero che esiste una differenza tra le città del Nord America, per esempio, e quelle del Sud Europa: oltre alle ovvie osservazioni empiriche, per rafforzare questa posizione 
basterebbe contrapporre alcuni teorici della città globale (SASSEN, 1991) o della post-metropoli (SOJA, 2000) ad altre voci che insistentemente, negli ultimi venti anni, evidenziano l'impossibilità di applicare i modelli occidentali a tutto il mondo (LEONTIDOU, 1990; 1996; BUNNEL e MARINGANTI, 2010; PARNELL e ROBINSON, 2012).

Durante il congresso della Association of American Geographers tenutosi a Los Angeles nel 2013, proprio Edward Soja, Allen Scott e Lila Leontidou si sono scontrati, in una sessione dedicata alle "Emerging Cities of the Third Wave" (SCOTT, 2011), sull'opportunità di utilizzare Los Angeles come paradigma di riferimento per gli studi urbani tout court. Mentre Soja, peraltro giunto a smorzare le sue teorie più radicali sulla post-metropoli in un'ottica più vicina all'urbanizzazione regionale (SOJA, 2011), concordava con Scott sulle lezioni che la città californiana può impartire al mondo intero, Leontidou tacciava i due esponenti della scuola di Los Angeles di colonialismo culturale, dovuto all'ignoranza delle specificità dell'Europa mediterranea.

In effetti molti studiosi di fatti urbani convergono ormai, negli ultimi anni, sul riconoscimento di differenze costitutive tra i vari modelli di città, e in particolare su ciò che distingue la città europea da quella nordamericana: P. Le Galès $(1998 ; 2003)$, per esempio, individua una specificità delle città europee risalendo, nella sua analisi dei modelli di governance, all'epoca medievale. Più recentemente e spostandosi a una scala ancor più sudeuropea, J. Seixas e A. Albet (2012) hanno analogamente riflettuto sulle caratteristiche della governance urbana nel "vero Mediterraneo", riprendendo una nota espressione di Braudel.

Questa convergenza di vedute rischia però di confondere alcuni aspetti. Prima di tutto, sussistono differenze evidenti anche tra le singole città dell'Europa mediterranea: per esempio, come si vedrà in seguito, a Palermo non si può parlare di una segregazione verticale come nel caso di Atene (MALOUTAS e KARADIMITRIOU, 2001), né allo ZEN i gruppi sociali più segregati sono identificabili per le loro origini etniche, come avviene in altri casi (ARBACI, 2008). Pertanto, nell'analizzare il caso del quartiere ZEN occorrerà considerare alcuni elementi peculiari della città siciliana, come le difficoltà della pianificazione in presenza di una forte criminalità organizzata (BONAFEDE e LO PICCOLO, 2010) o, viceversa, le pressioni esercitate da associazioni del "terzo settore" nell'attivare processi di coinvolgimento dal basso (NOVARA e VARVERI, 2015). Inoltre, per comprendere la segregazione socio-spaziale a Palermo sarà necessario tenere conto anche di altri cambiamenti in atto, come per esempio la proliferazione - estremamente tardiva per gli standard europei e nord-americani - di centri commerciali in città (TULUMELLO e PICONE, 2016).

Il secondo caveat su cui questo articolo insiste è il rischio del considerare le città dell'Europa mediterranea come un virtuoso esempio di informalità, in cui il welfare state e un certo familismo (amorale, direbbe E.C. BANFIELD, 1958) hanno sì ostacolato, da un lato, lo sviluppo economico e sociale, ma - paradossalmente - anche limitato lo strapotere neoliberista. Queste interpretazioni, per quanto affascinanti, devono comunque fare i conti con una realtà che, come vedremo, è molto più neoliberista di quanto non si pensi.

Le considerazioni fin qui esposte saranno quindi declinate sul tema della segregazione socio-spaziale, adottando un approccio teorico più ampio e aperto (OBERTI e PRÉTECEILLE, 2016) rispetto alla tradizione della scuola di Chicago (per un approfondimento degli studi sulla segregazione si rimanda a MASSEY e DENTON, 1993; KAPLAN, 2005). Rispetto a questa tradizione, spesso ancora legata a ormai classiche misurazioni quantitative del livello di integrazione delle minoranze etniche (DUNCAN e DUNCAN, 1955), il presente articolo affianca alle analisi quantitative anche alcune interpretazioni di natura qualitativa (DELYSER et alii, 2010). L'obiettivo sarà di mostrare come nel caso dello ZEN i pattern di segregazione (e de-segregazione) socio-spaziale si ripetano a diverse scale in maniera simile, mescolando elementi della classica segregazione di ispirazione nord-americana ad altre caratteristiche uniche del contesto locale.

\section{2 - Una geostoria in quattro movimenti}

Si potrebbe raccontare la storia dello ZEN (il cui acronimo sta per Zona Espansione Nord) suddividendola in tappe cronologiche, come ha fatto C. QUARTARONE (2008), oppure legandola alle politiche di social housing nel Sud Italia, come propone E. STELLA (2008), o ancora intercettando l'atto creativo dell'architetto più noto nella storia di questo quartiere, Vittorio Gregotti, come fa A. SCIASCIA (2003). $\mathrm{O}$, infine, si potrebbe tentare di spiegare come mai i mass media abbiano narrato fino all'esasperazione il degrado dello ZEN e di altri quartieri analoghi, tra cui le Vele di Scampia, replicando all'infinito una immagine stereotipata e stigmatizzante, come suggerisce F. FAVA (2007). Tuttavia, coerentemente con le premesse indicate all'inizio, di seguito intendo ricostruire una geostoria dello ZEN partendo da quattro luoghi in grado di descrivere quattro fasi storiche ben distinte e quattro diverse concezioni di segregazione.

Ciascuno di questi luoghi è allo stesso tempo concreto, perché fa riferimento a un contesto reale, e simbolico, perché assume un carattere esemplare nella geostoria dello ZEN. Ho scelto la parola "movimento" per indicare questi quattro luoghi, poiché la polisemia di questo termine, oltre a richiamare una composizione musicale, allude anche a un concetto geografico prima ancora che storico, un itinerario lungo il quale si sviluppa il tema della segregazione palermitana. I quattro movimenti che descriverò di seguito sono il baglio, il padiglione, l'insula $3 \mathrm{E}$ e il centro commerciale.

\section{I - II baglio}

Il primo movimento, dunque, è il baglio. Questa parola 
indica una tenuta agricola fortificata, edificata intorno a un ampio cortile centrale. Si tratta di una tipologia edilizia storica molto diffusa in Sicilia e ben presente all'interno della Piana dei Colli, l'area a nord di Palermo in cui sorge lo ZEN (fig. 1). Nel quartiere sopravvivono due bagli (Baglio Mercadante e Fondo Trapani), in forte stato di degrado: la Piana dei Colli, con le sue borgate agricole, è stata devastata, a partire dagli anni '50, da uno sviluppo edilizio sregolato, spesso sospinto dal legame tra criminalità ed edificazione (CANNAROZZO, 2009). Proprio al periodo tra il 1958 e il 1970 risale il primo nucleo di edificazione del quartiere, che si concentra intorno al Borgo Pallavicino e poi al cosiddetto ZEN 1, previsto in un PEEP (Piano di Edilizia Economica e Popolare) approvato nel 1966.

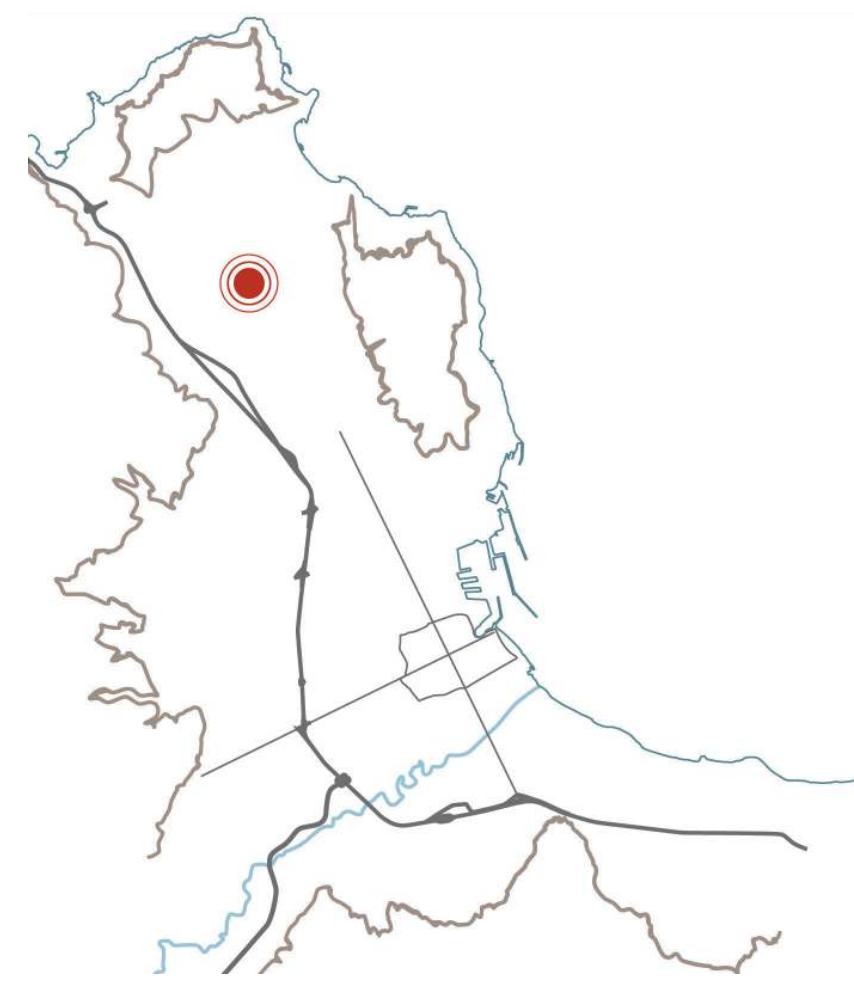

Fig. 1 - Localizzazione dello ZEN - Fonte: Disegno di Giancarlo Gallitano.

Ammesso di poter parlare di segregazione socio-spaziale per questo primo movimento, va detto che il baglio, retaggio di un passato contadino, non nasceva con lo scopo di rinchiudere la popolazione in un fortino inespugnabile, né le tipologie edilizie impiegate per Borgo Pallavicino e per lo ZEN 1 hanno attratto automaticamente gruppi sociali svantaggiati. Anzi, a voler giudicare oggi, a distanza di cinquant'anni, i risultati di questa prima fase, potremmo affermare che il nucleo composto da Borgo Pallavicino e ZEN 1, considerando lo stato di conservazione degli edifici, è in una condizione ottima: nel 2011, gli edifici in stato di conservazione mediocre o pessimo sono il 5,8 \% del totale degli edifici a Borgo Pallavicino e sono addirittura inesistenti allo ZEN 1, contro un valore del 48,1 \% allo ZEN 2 e una media del 16,6 \% nel Comune di Palermo. Semmai è nella localizzazione geografica che potremmo riscontrare tracce di una volontà segregante: lo ZEN 1 nasce circa 4 chilometri a nord-ovest di quelle che all'epoca erano le ultime propaggini periferiche urbane, nel mezzo di una zona che manteneva un forte carattere agricolo. Si trattava di un'operazione legata alla speculazione edilizia: l'idea, invero banale, di costruire un quartiere PEEP molto lontano dal nucleo urbano prefigurava la volontà di riempire gli spazi interstiziali con interventi privati, incuranti delle norme del Piano Regolatore Generale, dei requisiti legati ai servizi e del rispetto del tessuto urbano preesistente. Tuttavia, molti quartieri periferici palermitani (e, in genere, dell'Europa mediterranea) nascono per motivazioni analoghe, ma sono riassorbiti dallo sviluppo edilizio successivo, col risultato che la segregazione localizzativa diminuisce naturalmente man mano che la città ingloba queste periferie nel suo sviluppo.

La situazione cambia improvvisamente nel gennaio 1968, quando un forte terremoto colpisce la Sicilia occidentale e provoca il crollo di molte case del centro storico palermitano, peraltro già gravemente danneggiato dai bombardamenti alleati del 1943 e non ancora compiutamente riqualificato. Gli sfollati del centro storico, per lo più appartenenti alle classi sociali più deboli, richiedono al Comune di edificare nuove case; nel 1968 questo bandisce un concorso pubblico per l'edificazione di un ampliamento del quartiere ZEN, ampliamento che verrà in seguito conosciuto come ZEN 2 e che ci conduce al secondo movimento geostorico: il "padiglione".

\section{2 - II padiglione}

Il vocabolo padiglione è improprio, perché non si tratta di una tipologia edilizia riconosciuta. Più correttamente si dovrebbe parlare di insula, il termine scelto dal gruppo di architetti vincitori del concorso del 1968 per indicare l'unità abitativa che viene ripetuta, sempre uguale a se stessa, come elemento fondativo dello ZEN 2 (AMOROSO et alii, 1975). L'insula è ispirata a modelli architettonici del passato e richiama, per la sua forma, l'idea del baglio; naturalmente il debito culturale che i progettisti hanno nei confronti di Le Corbusier e del Movimento Moderno è palese, sia nelle forme sia nei materiali utilizzati, ma soprattutto nella concezione dello spazio. Il termine padiglione è impiegato al posto di insula quotidianamente dagli abitanti, che forse hanno colto "l'implicito riferimento, non solo dimensionale, alla fabbrica" (QUARTARONE, 2008: 263). Il padiglione è diventato l'immagine più nota e più ripetuta dell'intero ZEN, benché nei fatti appartenga solo allo ZEN 2: questa sineddoche ha appiattito e uniformato le differenze interne al quartiere, che in realtà esistono e sono profondamente tangibili.

Se la segregazione socio-spaziale dello ZEN 1 risiedeva più nella sua localizzazione geografica che in altro, in questo secondo movimento tutto parla, invece, di esclusione. La forma fisica del padiglione, con poche vie di accesso, con i marciapiedi rialzati, con lo spazio dei cortili interni frammentato da inopportune scale di accesso alle abitazioni, sembra davvero - e molto più del baglio storico - trasmettere l'idea di un fortino che si chiude al mondo esterno. La segregazione dello ZEN 2 passa anche dalla sua piazza centrale, che nel progetto di Gregotti e soci doveva contenere tutti i servizi per la cittadinanza, mai realizzati. Su questo punto progettisti e amministratori 
si rimpallano le colpe: i primi accusano la politica di non aver mai dotato il quartiere dei servizi previsti nel progetto (SCIASCIA, 2003), per scarsa volontà o, peggio, per mantenere una riserva di voti elettorali cui poter attingere facilmente, con la promessa costantemente disattesa di un miglioramento della qualità di vita. Tuttavia, è anche vero che altri quartieri analogamente progettati sono risultati similmente fallimentari, pur laddove i servizi fossero stati di fatto realizzati.

A marcare il confine tra ZEN 1 e ZEN 2 è la chiesa di San Filippo Neri (a questo santo, peraltro, è stato recentemente dedicato il quartiere, eliminando dalla nomenclatura ufficiale l'acronimo ZEN, sebbene ancor oggi tutti gli abitanti continuino a indicarlo alla vecchia maniera). La chiesa, costruita alla fine degli anni '90, si apre con un viale maestoso e alberato verso lo ZEN 1, mentre mostra un retro anonimo e del tutto impenetrabile verso la piazza degradata dello ZEN 2, quasi fosse poco più di un ennesimo "padiglione". Diventa pertanto il simbolo di un nuovo modo di intendere la segregazione: una modalità che contrappone, polarizzandole, inclusione (ZEN 1) ed esclusione (ZEN 2). Questo passaggio ci segnala una forte discontinuità tra la fase dei bagli e quella dei padiglioni: lo ZEN 1, già negli anni '90 ma ancor più negli ultimi quindici anni, si è integrato nel tessuto periferico palermitano, mentre lo ZEN 2 diventa un luogo in cui, prima ancora della violenza criminale di alcuni abitanti, è lo stesso spazio a mostrarsi feroce, condizionando in negativo le pratiche quotidiane (LO BOCCHIARO e TULUMELLO, 2014).

Nei due decenni in cui lo ZEN 2 viene realizzato (dal 1971 alla fine degli anni '80), la popolazione del quartiere arriva quasi a raddoppiare, mentre la popolazione complessiva della città inizia a subire, nel secondo decennio, un calo che diverrà sempre più cospicuo (tab. 1). A questo proposito, va detto che la popolazione dichiarata ufficialmente come residente allo ZEN non tiene conto di tutti gli occupanti abusivi, che negli anni si sono appropriati illegalmente delle abitazioni, soprattutto allo ZEN 2 (GIAMPINO, 2015), senza rispettare le liste di assegnazione gestite dal Comune e in forme talora molto conflittuali, che hanno indotto l'amministrazione a un immobilismo incapace di risolvere la questione del diritto alla casa. Le stime più realistiche indicano che la popolazione complessiva dello ZEN, sommando i residenti legali agli abusivi, superi ad oggi le ventimila unità.

Tab. 1 - Popolazione del quartiere ZEN (Unità di Primo Livello "San Filippo Neri") dal 1971 al 2011, per confronto con la popolazione complessiva del Comune di Palermo negli stessi anni

\begin{tabular}{|ccccc}
\hline ANNO & $\begin{array}{c}\text { POPOLAZ. } \\
\text { ZEN }\end{array}$ & VAR. \% & $\begin{array}{c}\text { POPOLAZ. } \\
\text { PALERMO }\end{array}$ & VAR. \% \\
\hline $\mathbf{1 9 7 1}$ & 7.239 & -- & 641.814 & -- \\
\hline $\mathbf{1 9 8 1}$ & 8.393 & $+15,9 \%$ & 701.765 & $+9,3 \%$ \\
\hline $\mathbf{1 9 9 1}$ & 13.315 & $+58,6 \%$ & 694.685 & $-0,9 \%$ \\
\hline $\mathbf{2 0 0 1}$ & 14.021 & $+5,3 \%$ & 686.722 & $-1,1 \%$ \\
\hline $\mathbf{2 0 1 1}$ & 15.004 & $+7 \%$ & 657.561 & $-4,2 \%$ \\
\hline
\end{tabular}

Fonti: de Spuches, Guarrasi, Picone, 2002: 166; http://gisportal.istat.it/ bt.viewer/
Un elemento importante per comprendere la segregazione allo ZEN è l'analisi della popolazione straniera presente all'interno del quartiere. Nei dati al 2011 (PICONE e SCHILLECI, 2012), la percentuale di popolazione straniera sul totale della popolazione è inferiore allo ZEN 1 (0,16 \%) e superiore allo ZEN 2 (4,66\%); si tratta comunque di numeri molto bassi, in linea con la scarsa incidenza della popolazione straniera a Palermo (2,99 \%). Questa considerazione induce pertanto a scartare a priori l'idea di una segregazione per lo più etnica nel quartiere, rifiutando quindi in questo caso le categorie descrittive proposte da S. ARBACI (2008). La segregazione, nel caso dello ZEN, non sembra dipendere da variabili come etnia, genere o generazione, ma altri elementi tracciano invece un quadro molto diverso: la percentuale dei laureati sulla popolazione totale allo ZEN è dell'1,33 \% (Palermo ha il 12,04 \%); anche considerando, oltre ai laureati, chi possiede un diploma di scuola superiore lo ZEN rimane a percentuali molto basse (11,48 \%, rispetto al 38,40\% di Palermo). Inoltre, il tasso di disoccupazione allo ZEN è del 16,88\%, rispetto alla media palermitana dell' $11,50 \%$, e il tasso di occupazione è di appena 25,50 \% (contro il 43,79 \% di Palermo). Si può parlare, pertanto, di una segregazione legata alla sfera educativa e a quella lavorativa.

Rispetto a tutte le variabili sin qui descritte, vi è sempre una differenza significativa tra lo ZEN 1, che si colloca in posizioni più vicine alla media comunale, e lo ZEN 2. Per esempio, la percentuale di laureati in alcune sezioni censuarie dello ZEN 1 supera il $18 \%$, mentre in quelle dello ZEN 2 non valica il $10 \%$. Analoghe considerazioni si potrebbero ripetere per gli indicatori economici.

Uno sguardo alle piramidi della popolazione (grafici 1-2) fa comprendere come lo ZEN 2 presenti una composizione delle età ancora fortemente piramidale, con molti giovani $\mathrm{e}$ pochi anziani, lontana dalla classica forma a botte assunta dalla popolazione dell'intero Comune di Palermo.

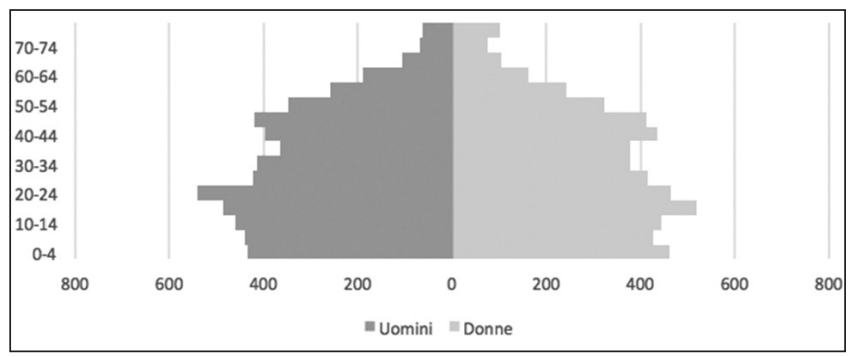

Graf. 1 - Piramide della popolazione dello ZEN 2

Fonte: Elaborazione dell'autore su dati ISTAT.

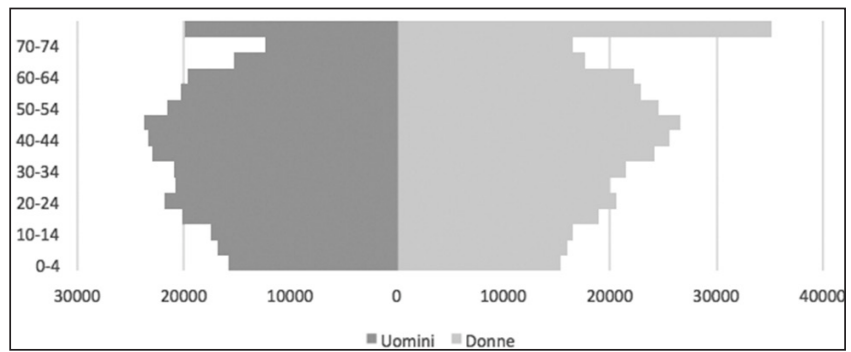

Graf. 2 - Piramide della popolazione del Comune di Palermo Fonte: Elaborazione dell'autore su dati ISTAT. 
La differenza tra ZEN 1 e ZEN 2 è marcata, quindi, da variabili socio-economiche; vi è tuttavia anche un fortissimo processo di stigmatizzazione mediatica' che plasma la storia dello ZEN 2 (de SPUCHES e PICONE, 2009) e che ha nel periodo tra il 1990 e il 2005 il suo apice, per poi lasciare il posto a un nuovo movimento geostorico, sintetizzabile nel racconto di quanto accade a un'insula particolare, la 3E.

\section{3 - L'insula 3E}

Nel progetto originario dello ZEN 2, le insulae erano indicate con un numero e una lettera, a partire da 0A. L'insula 3E non fu mai completata perché devastata da un incendio doloso (STELLA, 2008: 286) sviluppatosi durante uno dei periodi più bui del quartiere, verosimilmente per protesta contro la decisione del Comune di alloggiare in quest'insula un gruppo di cittadini rom. Al volgere del nuovo millennio, l'insula si presentava in uno stato di estremo degrado, sede di traffici illeciti, deposito di carcasse d'automobili, luogo simbolo dello sfacelo sociale e urbanistico del quartiere (foto 1). Nel 2004 il Comune avviava un progetto di recupero, che si completava nel 2010 (PITROLO, 2012); la nuova insula presenta una forma che è dotata, per la prima volta nella storia dello ZEN, di servizi pensati per la cittadinanza: una biblioteca di quartiere (mai inaugurata), uno sportello di consulenza per madri single, un asilo nido, un piccolo anfiteatro destinato a ospitare spettacoli e una caserma di carabinieri, avamposto delle forze dell'ordine nel quartiere. L'impatto visivo del cambiamento, grazie anche alla dotazione di verde pubblico nel cortile interno, è impressionante, e l'attuale insula $3 \mathrm{E}$, peraltro abitata solamente da assegnatari legittimi ${ }^{2}$, sembra un luogo fin troppo perfetto rispetto al resto del quartiere (foto 2 ).

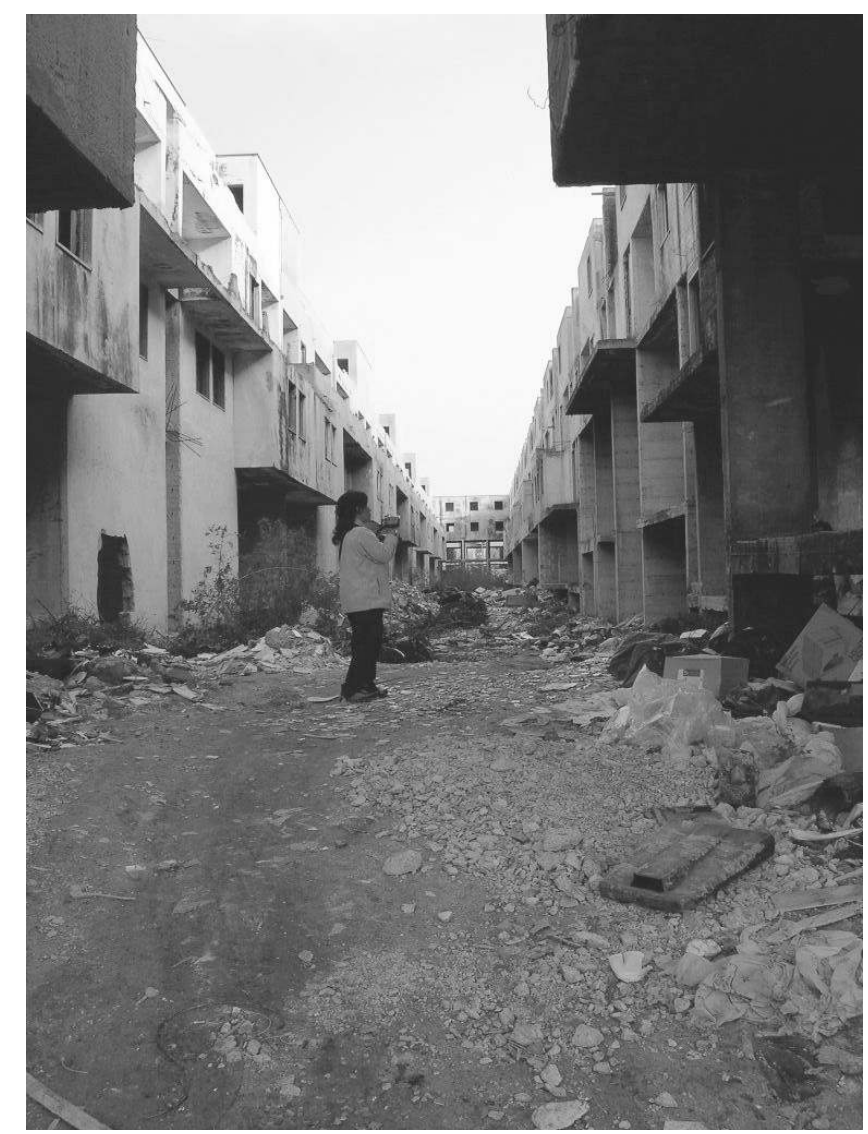

Foto 1 - L'insula 3E nell'aprile 2005 - Fonte: Stefania Sola, aprile 2005.

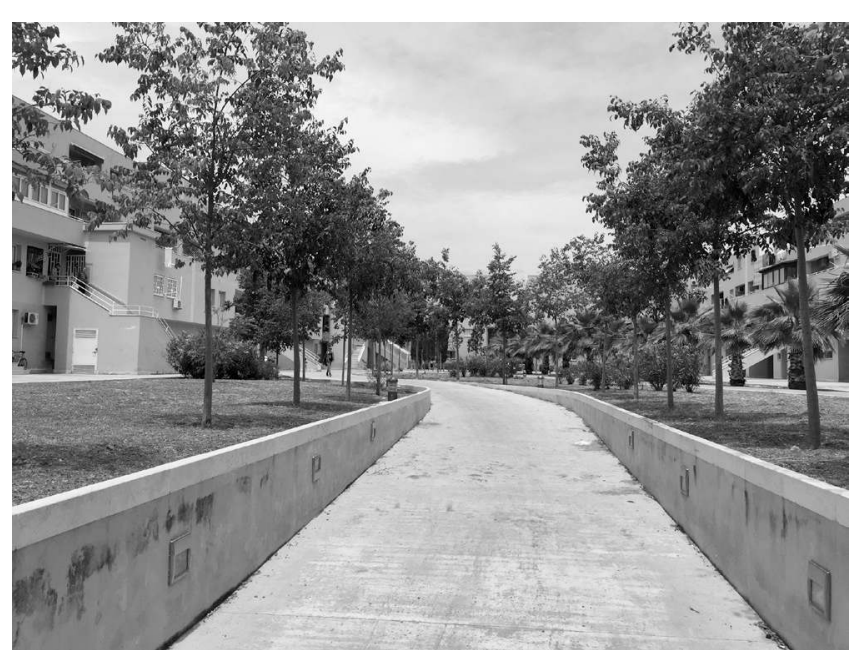

Foto 2 - L'insula 3E nel maggio 2016 - Fonte: Marco Picone, maggio 2016.

Si tratta apparentemente di un movimento de-segregante ${ }^{3}$ : indubbiamente la nuova insula è parte di un progetto più ampio, che mira a modificare l'immagine del quartiere e sottrarla agli sguardi stigmatizzanti;

docufilm diben diversa caratura. In Zenigma (2005), il regista Ar o presenta una visione ancora molto tradizionale del quartier con estratti di interviste a cittadini palermitani che, pur ignorando la posizione del quartiere, dimostrano di avere idee molto chiare rispetto alla sua (presunta) pericolosità (PICONE, 2011). CityZEN di Ruggero Gabbai (2015), invece, offre una lettura più complessa e attenta dello ZEN, decostruendo lo stereotipo negativo legato al quartiere e proponendo interpretazioni più sfumate, con scelte compositive estremamente efficaci.

2 Sarebbe interessante effettuare ulteriori analisi quantitative sulla percentuale di abitazioni occupate abusivamente o assegnate legittimamente, ma il Comune di Palermo non fornisce dati precisi in tal senso. Per una discussione più approfondita dei problemi del social housing a Palermo si rimanda a GIAMPINO, 2015.
3 L'intenzione del Comune di riportare il quartiere a condizioni di vita migliori è evidente nelle parole dell'allora sindaco Cammarata, che mirava a ribaltare "il vecchio concetto di isola esclusivamente residenziale e chiusa, [puntando] a creare un luogo aperto che sia un punto di riferimento nella vita del quartiere" (http://ricerca. repubblica.it/repubblica/archivio/repubblica/2005/05/17/un-piano"aprire" il quartiere verso la città, ovvero appunto di de-segregarlo. per-cambiare-lo-zen-meno.html). In altri termini, si pensava di 
dal punto di vista spaziale si tratta di un'opera di rigenerazione urbana in cui gli stessi abitanti hanno giocato un ruolo attivo [http://palermo.repubblica.it/ cronaca/2012/03/28/news/zen_gli_abusivi_risanano_l_ insula_questo_il_nostro_salotto-32319065/], ma molti intervistati (residenti e non) insinuano il sospetto che la presenza della caserma e il forte intervento comunale siano mirati a instaurare una sorta di "stato di polizia" (ROSSI e VANOLO, 2010) che garantisca un ordine più apparente che reale, quasi che gli enti pubblici volessero contenere la criminalità locale con la forza, anziché avviare processi educativi e partecipativi che agissero sul piano culturale. Le associazioni del terzo settore che lavorano all'interno del quartiere, ad esempio, hanno avuto un ruolo marginale nella riqualificazione dell'insula. È senz'altro vero che oggi l'insula $3 \mathrm{E}$ ha in qualche misura limitato le spinte alla segregazione socio-spaziale, ma occorrerebbe chiedersi se il modello di questa azione vada nella direzione corretta. Il processo di apparente de-segregazione legato a questo terzo movimento geostorico, infatti, non indica tanto un'apertura del quartiere verso l'esterno, né attira all'interno abitanti di gruppi sociali diversi, ma si limita, realisticamente, a creare una nuova enclave nell'enclave: un rifugio quasi paradisiaco all'interno di un quartiere che, nella percezione dei cittadini, è ancora un angolo d'inferno.

Paradossalmente, la de-segregazione voluta dal Comune finisce per creare una sorta di piccola gated community all'interno di un ghetto ${ }^{4}$ : non a caso infatti l'insula $3 E$ è ben isolata dal resto del quartiere, e vi si può accedere solo da un varco "protetto" dalla caserma. Nei fatti, è una specie di segregazione al quadrato.

\section{4 - II centro commerciale}

L'ultimo movimento geostorico è legato al centro commerciale Conca d'Oro, inaugurato il 13 marzo 2012. Palermo rappresenta un caso d'eccezione nel panorama dell'Europa mediterranea, perché fino al 2009 era probabilmente l'unica metropoli europea priva di centri commerciali (TULUMELLO e PICONE, 2016: 123). La notizia dell'inaugurazione del Conca d'Oro, cronologicamente terzo in città ma secondo per estensione $(55.000 \mathrm{mq})$, voluto dall'imprenditore Maurizio Zamparini (noto in città per essere il presidente della società calcistica locale), è stata accolta con grande entusiasmo dagli abitanti stessi dello ZEN. Il centro sorge a poche centinaia di metri dallo ZEN 1, ed è ben collegato con il quartiere. Nella percezione degli abitanti, confermata da numerose interviste, il centro commerciale ha colmato l'assenza di servizi e di luoghi d'incontro del quartiere, e soprattutto dello ZEN 2. In un contesto socialmente deprivato e pericoloso, assume una funzione salvifica che è stata definita "messianica"

\footnotetext{
4 Lo ZEN è stato a tutti gli effetti considerate un "ghetto" (FAVA, 2007) sia per motivi sociali (logiche matrimoniali, comunità chiuse, scarsi livelli di istruzione, ecc.) sia per ragioni geografiche: lo dimostra la rete stradale che circonda il quartiere e limita i suoi contatti con l'esterno.
}

(TULUMELLO e PICONE, 2016: 117), poiché gli abitanti dichiarano che finalmente qualcuno ha pensato a fornire loro un luogo positivamente connotato (moderno, pulito, accogliente) in cui trascorrere le giornate con le famiglie, tra shopping e divertimenti. Un luogo finora inesistente, poiché la piazza deputata a contenere i servizi, come si è visto, non è mai stata completata. Un luogo in cui i pericoli del mondo esterno vengono allontanati grazie a questa panacea, tempio del consumismo. In realtà, come è chiaro, il centro commerciale è un paradosso neoliberista e pseudo-pubblico (DAVIS, 2006), in cui gli imprenditori privati si avvantaggiano della crisi dell'attore pubblico per sottrarre spazi alla città e utilizzarli per fini privati e redditizi. Il debole contesto istituzionale palermitano (BONAFEDE e LO PICCOLO, 2010) ha creato le basi ideali affinché le politiche neoliberiste di Zamparini potessero attecchire in un'area parossisticamente fragile, tanto che l'imprenditore otteneva senza difficoltà nel 2006 una variante urbanistica per edificare il suo centro commerciale in una zona destinata dal Piano Regolatore a verde storico, promettendo in cambio di realizzare servizi per la cittadinanza (una scuola per ipovedenti, una piscina, campi di calcio, ecc.) ma non consegnando mai, nei fatti, tali strutture al Comune.

Anche questo quarto e ultimo movimento geostorico appare de-segregante, ma in modo del tutto diverso dal precedente: laddove l'insula 3E finiva per essere iper-segregante poiché creava una gated community in miniatura all'interno del ghetto, il centro commerciale apre lo ZEN a una dimensione nuova, in cui i flussi commerciali cittadini per la prima volta penetrano nel quartiere "infernale" (FAVA, 2007), o quanto meno lo lambiscono. Inoltre, il mall di Zamparini realizza luoghi pseudo-pubblici di aggregazione a due passi dallo ZEN, supplendo alle incapacità del Comune di offrire spazi per la socialità. E tuttavia, l'aspirazione de-segregante è fortemente filtrata da interessi commerciali che, in virtù delle logiche neoliberiste che le sorreggono, creano una situazione paradossale: è proprio il dogma globalizzante, è proprio il culto del commercio neoliberista a indicare generosamente una via di salvezza per il quartiere segregato. Esattamente l'opposto di quel che capita in altri contesti geografici, a partire da quello nord-americano. Non vi è stata, nel caso dello ZEN, una mobilizzazione contro il megaprogetto neoliberista: piuttosto che dimostrarsi un quartiere "ribelle" (HARVEY, 2012), di fronte alla prospettiva di modernizzarsi con un centro commerciale lo ZEN ha abbracciato in pieno il credo dello shopping in salsa sud-europea.

\section{3 - Mappare la segregazione allo ZEN}

Come si è visto nella sezione precedente, ai quattro movimenti geostorici indicati (baglio, padiglione, insula 3E, centro commerciale) corrispondono quattro concezioni diverse di segregazione: la prima è una segregazione localizzativa, tipica della stagione di edilizia popolare postbellica; la seconda è una stigmatizzazione mediatica, 
rinforzata dalla scelta di forme architettoniche generatrici di esclusione, che spezza il quartiere in due parti e le polarizza socio-spazialmente; la terza è una (apparente) de-segregazione che rischia di scivolare in uno stato di polizia e che in effetti fallisce nei suoi intenti; infine, la quarta è una de-segregazione paradossalmente imposta da dettami neoliberisti e trainata da un megaprogetto imprenditoriale.

Il passaggio successivo che questo articolo propone è una riflessione su come rappresentare cartograficamente i processi di segregazione in questo quartiere. Se si vuole superare l'idea stereotipata dello ZEN come luogo del degrado, di quartiere irredimibile, occorre moltiplicare le narrazioni e dar spazio a una polifonia di voci (PICONE, 2011); si può conseguire questo obiettivo soltanto utilizzando i metodi qualitativi, che, applicati agli studi urbani, mirano a descrivere un luogo alla luce di punti di vista differenti (SUI e DELYSER, 2012). I presupposti teorici di questo passaggio rientrano nell'alveo del pensiero ermeneutico e utilizzano il metodo induttivo (DELYSER et alii, 2010), partendo quindi dall'analisi delle specificità del caso studio per poi enuclearne principi validi in generale. Le rappresentazioni cartografiche scelte, pertanto, saranno di natura puramente qualitativa: ideogrammi e mappe mentali (LYNCH, 1960; GREGORY, 1994; DRIVER, 2005). Naturalmente si tratterà di un'interpretazione soggettiva e discutibile, ma ritengo che possa contribuire a "scendere in profondità" nelle analisi urbane ${ }^{5}$.

Nel tentativo di graficizzare la segregazione allo ZEN, sono partito dalla ricerca di figure geometriche che potessero rappresentare i quattro movimenti geostorici, scegliendo il cerchio come simbolo segregante e il cuneo (una punta di freccia) come simbolo de-segregante: il cerchio rappresenta la chiusura, l'enclave, il fortino; il cuneo simboleggia invece l'elemento di penetrazione che riduce o attenua la segregazione, creando un ponte tra esterno ed interno.

I quattro movimenti del caso ZEN, quindi, potrebbero essere rappresentati in un ideogramma come in fig. 2 . Schematicamente, qui, all'inizio lo ZEN è un grande cerchio (movimento 1: il baglio) isolato dal resto della città. In questo cerchio si insinua poi un cuneo de-segregante, legato alla "normalizzazione" dello ZEN 1 per contrapposizione alla stigmatizzazione dello ZEN 2

\footnotetext{
5 Dal punto di vista metodologico, le mappe mentali e gli ideogrammi presentati di seguito sono il frutto della percezione dell'autore e della sua interpretazione della segregazione, piuttosto che del sentire degli abitanti. Tuttavia, in difesa di questa posizione va esplicitato che le mappe mentali dello ZEN derivano da un ormai decennale lavoro sul campo, basato su tecniche qualitative come l'osservazione partecipante e le interviste (PICONE, 2011), e quindi dall'interazione diretta con gli abitanti. Inoltre, rispetto al lavoro apripista di K. Lynch, 1960 è necessario, a più di mezzo secolo di distanza, ripensare allo statuto teorico e operativo delle mappe mentali, ampliandone le categorie per abbracciare nuove impostazioni metodologiche (ALAIMO e PICONE, 2015).
}

(movimento 2: il padiglione). Questo cuneo esprime il massimo potenziale nel punto in cui si insedia la chiesa di San Filippo Neri (il cuneo più scuro), marcando un punto di frattura tra l'inclusione dello ZEN 1 e l'esclusione dello ZEN 2, che si racchiude in un cerchio ancor più segregato (rappresentato simbolicamente con un grigio più scuro).

Nella terza fase (movimento 3: l'insula 3E) anche all'interno dello ZEN 2 s'inserisce un piccolo cuneo de-segregante: si tratta della riqualificazione dell'insula 3E, che tenta di spezzare l'isolamento del quartiere. In ultimo (movimento 4: il centro commerciale), invece, il megaprogetto di Zamparini introduce un altro significativo cuneo.
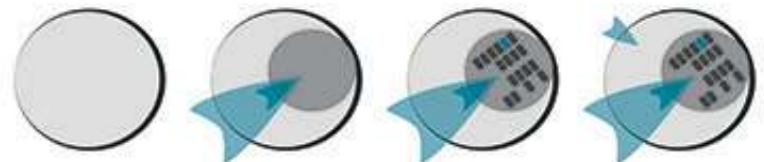

Fig. 2 - Ideogramma della segregazione e de-segregazione allo ZEN. 1: baglio; 2: padiglione; 3: insula 3E; 4: centro commerciale - Fonte: Disegno di Giovanna Ceno.

Il quadro d'insieme di questi quattro movimenti ci fornisce una prima mappa della segregazione allo ZEN (fig. 3). Qui ritroviamo lo schema ideogrammatico della fig. 2, ma questa volta il disegno assume una caratterizzazione più cartografica, divenendo una vera e propria mappa mentale. I pattern lineari o quadrettati indicano le diverse zone del quartiere; le linee perimetrali sono più o meno spesse in base al grado di isolamento che creano tra il quartiere e l'esterno; le linee tratteggiate indicano le vie di accesso allo ZEN; i cunei (più o meno sfumati a seconda del grado di de-segregazione a loro associato) individuano invece le porosità del quartiere, ovvero quei luoghi in cui il mondo esterno penetra più facilmente dentro lo ZEN.

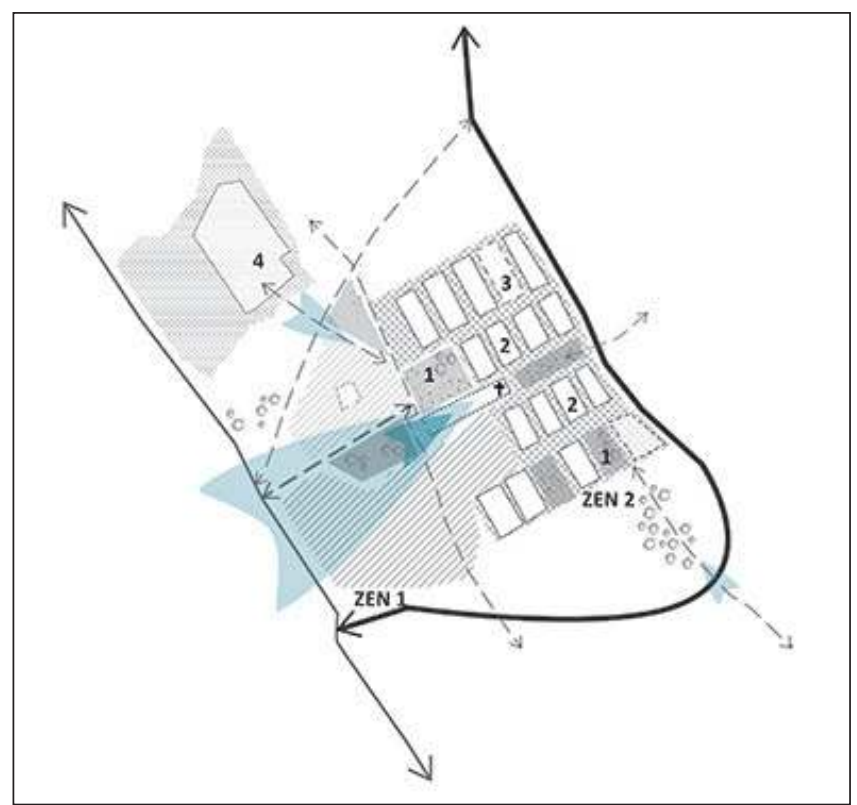

Fig. 3 - Mappa mentale schematica della segregazione e de-segregazione allo ZEN. 1: bagli; 2: padiglioni; 3: insula 3E; 4: centro commerciale Fonte: Disegno di Giovanna Ceno. 
Per disegnare una seconda mappa mentale, più dettagliata e meno schematica, ma pur sempre frutto del modo in cui la segregazione può essere percepita attraverso tecniche qualitative si è deciso di partire dalla carta tecnica comunale, adottando una visuale a volo d'uccello e poi disegnando in maniera volutamente incurante delle regole geometriche di rappresentazione cartografica, per rispecchiare piuttosto il punto di vista del flâneur. Il risultato è una rappresentazione complessa del quartiere (fig. 4), che individua aree preesistenti (legate dunque ai bagli), il nucleo dello ZEN 2 (l'area dei padiglioni), il centro commerciale come nuovo legame con l'esterno, le scuole (elementi di presidio pubblico all'interno del quartiere) e quei cunei di penetrazione de-segregante di cui si è già detto sopra. In un dettaglio dello ZEN 2 (fig. 5), poi, l'insula $3 \mathrm{E}$ emerge come elemento di rinnovamento in un tessuto generalmente degradato.

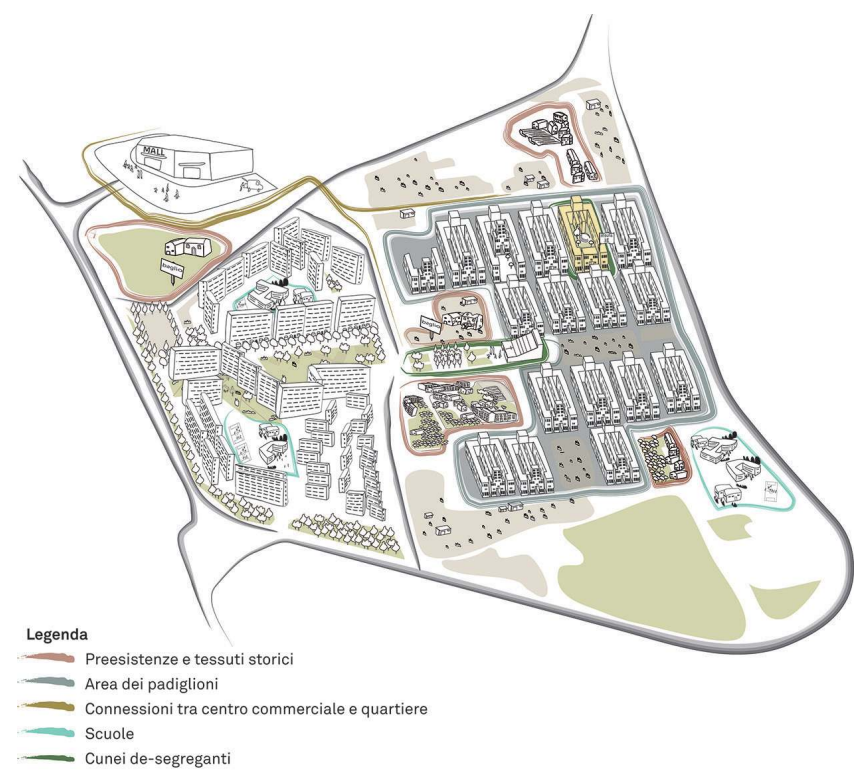

Fig. 4 - Mappa mentale dettagliata del quartiere ZEN - Fonte: Disegno di Giancarlo Gallitano.

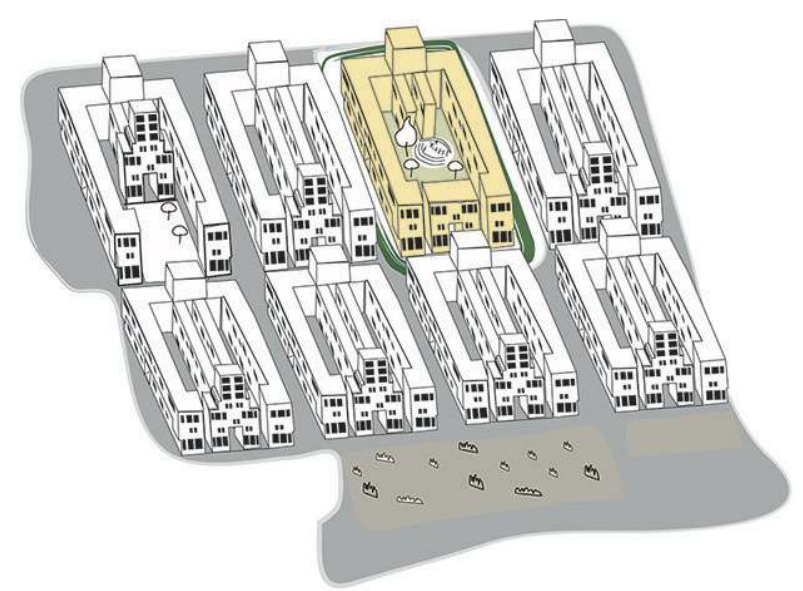

Fig. 5 - Dettaglio dello ZEN 2 - Fonte: Disegno di Giancarlo Gallitano.

Le mappe e gli schemi qui presentati mostrano come la segregazione allo ZEN sia un tema complesso e non riassumibile in una banale ghettizzazione del quartiere rispetto alla città. Queste immagini sono il risultato di come oggi è possibile percepire quasi sessant'anni di continue azioni sul territorio, di flussi di abitanti che si spostano e di attori sociali pubblici e privati che, in modi spesso poco coerenti, tentano di perseguire i propri interessi, siano essi rivolti alla collettività o meno. L'ultimo passaggio da compiere è il tentativo di trarre indicazioni più generiche dalla geostoria di questo quartiere palermitano.

\section{4 - Conclusioni: un modello paradossale e multi-scalare}

A partire dalle osservazioni e rappresentazioni raccolte, questa sezione conclusiva intende fornire alcune risposte alla questione della segregazione allo ZEN, riprendendo le domande poste nelle sezioni precedenti. Che cosa può insegnare la lezione dello ZEN a chi si occupa di segregazione nelle città dell'Europa mediterranea?

Innanzitutto, si è notato che la segregazione allo ZEN è stata mitigata da varie azioni de-segreganti (i cunei), ed è facile verificare che, nei fatti, il quartiere non ha mai raggiunto indici di segregazione elevatissimi, quanto meno nel caso dello ZEN 1, come mostrano i dati demografici. Inoltre, la segregazione etnica è pressoché del tutto assente. Si tratta di una caratteristica tipica delle città mediterranee? Sicuramente le reti di relazioni familiari strette, ma anche la forte azione delle associazioni e le dinamiche di spostamento legate al terremoto del 1968 hanno conservato forti legami tra i residenti dello ZEN e gli abitanti di altre aree della città, come il centro storico o le borgate ormai inserite entro il tessuto urbano.

Benché si possa riscontrare un certo livello di omogeneità sociale, è difficile considerare oggi lo ZEN nel suo complesso come un ghetto che racchiude un unico gruppo sociale deprivato, anche nel caso dello ZEN 2, dove la distinzione tra abitanti abusivi e legittimi crea gruppi facilmente riconoscibili. In questo senso, ritengo che sia ravvisabile una specificità mediterranea che attenua le tendenze segreganti diffuse nelle città nord-americane o nord-europee, in virtù di politiche di welfare e interventi comunali che mirano ad attenuare il divario sociale.

Semmai va evidenziata una tendenza molto specifica del caso di studio: laddove il neoliberismo avanza e i soggetti pubblici perdono il loro potere d'intervento, si può asserire che il risultato è sempre negativo e aumenta la segregazione, come racconta la letteratura (ARBACI, 2008; HARVEY, 2012)? In quest'ottica, ritengo che lo ZEN segni un'eccezione notevole: i megaprogetti neoliberisti e i fallimenti delle politiche comunali, come si è visto, hanno paradossalmente colmato - in un sistema fragile e dotato di un vago concetto di cosa sia o debba essere pubblico - un vuoto, riducendo la segregazione percepita del quartiere mediante due inserimenti molto diversi tra loro (la caserma e il centro commerciale) che in altri contesti, a partire da quelli nord-americani, avrebbero probabilmente generato una rivolta della popolazione e che a Palermo, invece, sono stati accolti come salvifici. I meccanismi 
che regolano l'esclusione sociale nel capoluogo siciliano, quindi, sono solo in parte assimilabili a quelli che valgono per le città degli USA o dell'Europa settentrionale, dove la globalizzazione neoliberista aumenta gli effetti di polarizzazione sociale che qui, invece, sembra bilanciata proprio dalle influenze di un neoliberismo tardivo e incompleto, quasi che la crisi economica esplosa nel 2008 stia riequilibrando dinamiche polarizzanti.

Un altro punto da appurare è se la segregazione, in questa particolare città mediterranea, segua o meno una logica spaziale riconoscibile. Ritengo che la logica spaziale tipica dello ZEN sia multi-scalare: come si è visto, infatti, nel quartiere si creano distinzioni tra luoghi più o meno segregati, in un continuo rimescolamento tra inclusione ed esclusione.

In una prima fase (movimento) lo ZEN è un'isola dispersa in mezzo a campi agricoli, luogo in cui si raccolgono gli sfollati del centro storico e altri gruppi socialmente deboli: un macro-livello di segregazione spaziale.

In seguito (secondo movimento), lo ZEN 2 assume tutte le connotazioni più stigmatizzate e segreganti, mentre lo ZEN 1 inizia la sua rincorsa alla normalità: qui si marca uno spostamento verso un meso-livello in cui la segregazione socio-spaziale non coincide più con l'intero quartiere ma con una sua parte, che però diviene esemplificativa del tutto (la sineddoche di cui sopra).

Anche dentro lo ZEN 2, però, i movimenti più recenti (vedi il caso dell'insula 3E) mostrano che non è possibile parlare di una segregazione tout court, che coinvolga il quartiere nella sua interezza: ci sono luoghi più segregati, come i padiglioni occupati dagli abusivi, e luoghi che invece incoraggiano un'apertura verso l'esterno, come l'insula $3 \mathrm{E}$. A questo micro-livello, quindi, la segregazione riguarda solo alcuni padiglioni.

Si tratta di un modello che si potrebbe definire di "segregazione a matrioska": il macro-, il meso- e il microlivello funzionano allo stesso modo, pur se a diverse scale, poiché creano una contrapposizione facilmente riscontrabile tra aree fortemente segregate ed altre che sono invece ben connesse con le dinamiche urbane palermitane, proprio come accade in una matrioska, in cui ogni bambola più piccola ricalca esattamente la forma di quella più grande.

I due elementi fin qui richiamati, ovvero il paradosso di un neoliberismo de-segregante e la logica multi-scalare a matrioska, a mio avviso costituiscono le caratteristiche proprie della segregazione allo ZEN, e in generale (pur con alcune differenze) del caso di Palermo. Si tratta di specificità locali che però credo possano essere assunte come caratteristiche di rottura rispetto al modello di segregazione nord-americana, e che probabilmente affondano le loro radici nella mediterraneità del contesto. Le intuizioni che partono dal caso ZEN possono dunque servire a instillare qualche dubbio in quegli studiosi che ritengono di poter raccontare un'unica storia, universale e globalizzata, per spiegare la segregazione socio-spaziale nel mondo.

\section{Ringraziamenti}

L'autore ringrazia Giovanna Ceno e Giancarlo Gallitano per aver realizzato gli ideogrammi e le mappe mentali della segregazione allo ZEN. Ringrazia inoltre Thomas Pfirsch e Giulia de Spuches per averlo stimolato a raccogliere le sue riflessioni sul quartiere.

\section{Riferimenti bibliografici}

ALAIMO A. e PICONE M., (2015), Shadowing and Qualitative Gis: Tools for Urban Narrations, Scienze del Territorio, vol. 2, n³, p.391-399.

AMOROSO F., BISOGNI S., GREGOTTI V., MATSUI H. e PURINI F., (1975), Quartiere Zen a Palermo, Lotus International, vol. 9, nº 1, Industrie Grafiche Editoriali, Milano, p. 6-27.

ARBACI S., (2008), (Re)Viewing Ethnic Residential Segregation in Southern European Cities: Housing and Urban Regimes as Mechanisms of Marginalisation, Housing Studies, vol. 23, n 4, p. 589-613.

BANFIELD E. C., (1958), The Moral Basis of a Backward Society, Free Press, New York, p. 188.

BONAFEDE G. e LO PICCOlO F., (2010), Participative Planning Processes in the Absence of the (Public) Space of Democracy, Planning Practice and Research, vol. 25, n 3, p. 353-375.

BUNNEL T. e MARINGANTI A., (2010), Practising Urban and Regional Research beyond Metrocentricity, International Journal of Urban and Regional Research, vol. 34, n², p. 415-420.

CANNAROZZO T., (2009), La governance mafiosa e l'assalto al territorio, in LEONE M., LO PICCOLO F. e SCHILLECI F. (a cura di), Il paesaggio agricolo nella Conca d'Oro di Palermo, Alinea, Firenze, p. 39-51.

DAVIS M., (2006), City of Quartz: Excavating the Future in Los Angeles, Verso, London, p. 462.

DELYSER D., HERBERT S., AITKEN S., CRANG M., MCDOWELL L. (a cura di), (2010), The Sage Handbook of Qualitative Geography, Sage, London, p. 448.

DRIVER F., (2005), Imaginative Geographies, in CLOKE P., CRANG P., GOODWIN M. (a cura di), Introducing Human Geographies, Routledge, London, 2005, p. 209-216.

DUNCAN O. D. e DUNCAN B., (1955), Residential Distribution and Occupational Stratification, American Journal of Sociology, vol. 60, $\mathrm{n}^{\circ} 5$, p. 493-503. 
FAVA F., (2007), Banlieue de Palerme. Une version sicilienne de l'exclusion urbaine, L'Harmattan, Paris, p. 384.

GIAMPINO A., (2015), Lo spazio conteso tra pratiche informali e processi formali, Urbanistica informazioni, vol. 42, n 261-262, p. 55-57.

GREGORY D., (1994), Geographical Imaginations, Blackwell, Cambridge, p. 456.

HARVEY D., (2012), Rebel Cities: From the Right to the City to the Urban Revolution, Verso, London, p. 206.

KAPLAN D.H., (2005), Research in Ethnic Segregation II: Measurements, Categories and Meanings, Urban Geography, vol. 26, n 8, p. 737-745.

LE GALÈS P., (1998), La nuova “political economy” delle città e delle regioni, Stato e Mercato, vol. 9, n 1, p. 53-92.

LE GALÈS P., (2003), European Cities. Social Conflict and Governance, Oxford University Press, Oxford, p. 328.

LEONTIDOU L., (1990), Urban Social Movements in 'Weak' Civil Societies: The Right to the City and Cosmopolitan Activism in Southern Europe, Urban Studies, vol. 47, nº 6, p. 1179-1203.

LEONTIDOU L., (1996), Alternatives to Modernism in (Southern) Urban Theory: Exploring In-Between Spaces, International Journal of Urban and Regional Research, vol. 20, n², p. 178-195.

LO BOCCHIARO G. e TUlUMELlo S., (2014), La violenza dello spazio allo Zen di Palermo. Un'analisi critica sull'urbanistica come strumento di giustizia, Archivio di Studi Urbani e Regionali, vol. 45, n 110, p. 73-94.

LYNCH K., (1960), The Image of the City, MIT Press, Cambridge, p. 202.

MALOUTAS T. e KARADIMITRIOU N., (2001), Vertical Social Differentiation in Athens: Alternative or Complement to Community Segregation?, International Journal of Urban and Regional Research, vol. 25, nº 4, p. 699-716.

MASSEY D. S. e DENTON N. A., (1993), American Apartheid: Segregation and the Making of the Underclass, Harvard University Press, Cambridge, p. 312.

NOVARA C. e VARVERI L. (a cura di), (2015), Piazza comunità connessioni. Creazione di processi partecipativi, Aracne, Roma, p. 208.

OBERTI M. e PRÉTECEILLE E., (2016), La ségrégation urbaine, La Découverte, Paris, p. 124.

PARNELL S. e ROBINSON J., (2012), (Re)theorizing Cities from the Global South: Looking Beyond Neoliberalism, Urban Geography, vol. 33 , $\mathrm{n}^{\circ}$ 4, p. 593-617.

PICONE M., (2011), Lo Zen e l’arte della narrazione dei luoghi, Rivista Geografica Italiana, vol.118, n4, p.675-690.

PICONE M. e SCHILLECI F., (2012), Quartiere e identità. Per una rilettura del decentramento a Palermo, Alinea, Firenze, p.320.

PITROLO G., (2012), Il recupero dell'insula 3E allo ZEN 2: una buona pratica, in ALAIMO G. (a cura di), Lo ZEN 2 di Palermo: un laboratorio per il progetto e la gestione del recupero, Aracne, Roma, p. 119-130.

QUARTARONE C., (2008), Lo Zen a Palermo. La de-costruzione di un nucleo urbano autosufficiente, in BADAMI A., PICONE M. e SCHILLECI F. (a cura di), Città nell'emergenza. Progettare e costruire tra Gibellina e lo Zen, Palumbo, Palermo, p. 257-270.

ROBINSON J., (2006), Ordinary Cities. Between Modernity and Development, Routledge, London, p. 224.

ROBINSON J., (2011), Cities in a World of Cities: The Comparative Gesture, International Journal of Urban and Regional Research, vol. 35 , $\mathrm{n}^{\circ} 1$, p. $1-23$.

ROSSI U. e VANOLO A., (2010), Geografia politica urbana, Laterza, Roma-Bari.

SASSEN S., (1991), The Global City: New York, London, Tokyo, Princeton University Press, Princeton, p. 480.

SCIASCIA A., (2003), Tra le modernità dell'architettura. La questione del quartiere Zen 2 di Palermo, L'Epos, Palermo, p. 220.

SCOTT A.J., (2011), Emerging Cities of the Third Wave, City, vol. 15, n 3-4, p. 289-321.

SEIXAS J. e ALBET A. (a cura di), (2012), Urban Governance in Southern Europe, Ashgate, London, p. 256.

SOJA E., (2000), Postmetropolis. Critical studies of cities and regions, Blackwell, Oxford, p. 440.

SOJA E., (2011), Regional urbanization and the end of the metropolis era, in BRIDGE G. e WATSON S. (a cura di), The New Blackwell Companion to the City, Blackwell, Oxford, p. 679-689.

SPUCHES (de) G., GUARRASI V. e PICONE M., (2002), La città incompleta, Palumbo, Palermo, p. 240.

SPUCHES (de) G. e PICONE M., (2009), Stigma, in DI BIAGI P. e MARCHIGIANI E. (a cura di), Città pubbliche. Linee guida per la riqualificazione urbana, Bruno Mondadori, Milano, p. 232-235.

STELLA E., (2008), Zen, ovvero la città a pezzi. Problemi di integrazione della periferia, in BADAMI A., PICONE M. e SCHILLECI F. (a cura di), Città nell'emergenza. Progettare e costruire tra Gibellina e lo Zen, Palumbo, Palermo, p. 279-290.

SUI D., DELYSER D., (2012), Crossing the Qualitative-Quantitative Chasm I: Hybrid Geographies, the Spatial Turn, and Volunteered Geographic Information (VGI), Progress in Human Geography, vol. 36, n 1, p. 111-124.

TULUMELLO S. e PICONE M., (2016), Shopping Malls and Neoliberal Trends in Southern European Cities: Post-Metropolitan Challenges for Urban Planning Policy, Finisterra, vol. 51, n 101, p. 111-132. 